\title{
Articulaciones de edad y género en la construcción de feminidades en un programa de prevención del delito juvenil ${ }^{*}$
}

\author{
MARINA MEDAN**
}

\section{Resumen}

Este artículo analiza el interjuego existente entre las dimensiones de género y edad en la construcción de beneficiarias en un programa de prevención del delito juvenil implementado en Buenos Aires. El argumento se inscribe dentro de las discusiones que sostienen el rol central del Estado en la construcción del par inclusión/exclusión social. En el trabajo se reconstruye, primeramente, cómo un dispositivo estatal -originalmente pensado para varones- justifica la inclusión de chicas, generizando la situación de riesgo en la que ellas se encontrarían. Seguidamente, señala las consecuencias que dicho proceso tiene sobre la configuración de las feminidades juveniles. Los resultados indican que la legitimación institucional de necesidades de las beneficiarias condiciona la configuración de feminidades y restringe aspectos de la experiencia juvenil y femenina. No obstante, dicho proceso no representaría, desde el punto de vista de las chicas, una contrariedad.

Palabras clave: programa social - prevención del delito - juventud desfavorecida - género - edad

\begin{abstract}
This article analizes the interplay between gender and age in the female construction that takes place on a crime prevention programme set in Buenos Aires. The argument is inscribed within discussions about the central role that State plays in both social exclusion and inclusion. Firstly, the article reconstructs how a male orientated programme justifies the inclusion of girls, gendering the risk situations in which they would meet. Secondly, the article states what kind of impact that process has on young femininities. The results show that while the programme legitimizes girls needs, it also restricts juvenile and female experience. However, this process would not be seen as a contrariness by youth women.
\end{abstract}

Key words: social program - crime prevention - disadvantaged youth - gender - age

Medan, Marina "Articulaciones de edad y género en la construcción de feminidades en un programa de prevención del delito juvenil", en Zona Franca. Revista del Centro de Estudios Interdisciplinario sobre Mujeres, Año XXII, Nº 23, 2014, pp. 28-36.

Recibido: 12 de agosto 2014 - Aceptado: 2 de octubre 2014 


\section{Introducción}

ए I trabajo discute las articulaciones entre las dimensiones de edad y género que moldean la construcción de beneficiarias en un programa de prevención del delito juvenil implementado en el Gran Buenos Aires. Se analiza el tratamiento que este dispositivo estatal destinado a jóvenes $-y$ pensado para varones- realiza sobre las chicas. Los resultados sugieren que la legitimación institucional de necesidades de las beneficiarias condiciona la configuración de feminidades y restringe aspectos de la experiencia juvenil y femenina. No obstante, dicho proceso no representaría, desde el punto de vista de las chicas, una contrariedad. El argumento central se inscribe en las discusiones sobre la construcción social del par inclusión/exclusión, entendido ampliamente. Es decir, no sólo incluyendo en su configuración las desigualdades de clase social, sino aquellas producidas por particulares anudamientos de género y edad. En términos generales, intenta aportar a las descripciones y comprensiones sobre los modos de gobernar a la "juventud en riesgo" a partir de programas estatales, desde una perspectiva constructivista. ${ }^{1}$

Los programas sociales dirigidos a la infancia, adolescencia y juventud suelen definir su población destinataria otorgando centralidad a la dimensión etaria, a la que agregan condicionalidades de clase, etnia, o de género, y alguna posición respecto a una situación de riesgo que amerita la intervención. ${ }^{2}$ Asimismo, muchas de estas intervenciones suponen que su rol en la gestión de dicha situación de riesgo es colaborar en que los y las jóvenes construyan un proyecto de vida. Este objetivo sugiere que estas poblaciones no pueden hacerlo por sí solas y que necesitan asistencia para concretarlo; o que si acaso pueden hacerlo, la ayuda institucional redundará en que aquel que gesten sea un proyecto de vida "deseable". Ahora bien, mientras este proyecto de vida suele vincularse con trayectorias educativas y/o inserciones laborales sostenidas y la conformación de la familia propia, el artículo argumenta que éste no tiene las mismas características según se trate de chicas o varones. Esto porque la situación de riesgo que se gestionaría mediante el proyecto de vida no es la misma para varones y mujeres. Este proceso responde a que los programas no sólo colaboran con la construcción social y el procesamiento de las edades de las personas. Además, portan ideas sobre el género y sobre qué aspectos deberían caracterizar uno proyecto de vida deseable para chicas y para varones. A su vez, cuando ellas y ellos se encuentran con los programas se ponen en diálogo las expectativas y activos de ambas partes. ${ }^{3}$

El Estado, los programas sociales y las marcas de edad y género. Las intervenciones institucionales (ya sean estatales, religiosas, privadas, etc.) contribuyen, con sus definiciones de actores y objetivos, a la organización y caracterización de distintos grupos de personas, con fines de regulación social. Valeria Llobet (2009) sostiene que las instituciones cumplen un papel legislador al señalar las cláusulas bajo las que, por ejemplo, la inclusión social y cultural podrá darse, incluyendo el proceso de adquisición y reconstrucción de características típicas, el manejo de las relaciones sociales y su trans- formación en posiciones subjetivas singulares. En sus niveles programáticos hasta en sus implementaciones cotidianas los programas definen escenarios de riesgo sobre los cuales intervenir, interpretan necesidades de sus destinatarios, establecen satisfactores para ellas y legitiman las demandas que coinciden con esas interpretaciones; aún concibiendo dentro de ellos procesos heterogéneos y no siempre coherentes, los programas se establecen como la voz autorizada para definir las reglas del juego, los posibles y los impensables (Fraser 1991).

Cuando las poblaciones a regular son las nuevas generaciones, la tarea legislativa es aún más sensible. Se trata de garantizar el orden pero también la reproducción social (Llobet 2009). En ese punto, la transición entre la juventud y la adultez cobra un espacio clave y resulta una preocupación para el entramado estatal. La habitual definición de las situaciones de riesgo en los programas para jóvenes, premisa de las intervenciones, se vincula con la posibilidad de que determinada transición deseable no pueda concretarse. De allí la confianza de los programas en que la elaboración de un proyecto de vida pueda evitar desenlaces indeseables. ${ }^{4}$

La necesidad de armar un "proyecto" connota que carecer de él es un déficit que debe compensarse, al tiempo que señala, además, el carácter incompleto de la persona. Esta interpelación de los programas coloca a los beneficiarios/as como sujetos carentes (Chaves 2005), ubicados/as en una posición de menor jerarquía y evolución respecto de ciertos parámetros adultocráticos en torno a la conformación plena de una persona. En esa preocupación 
transicional, la edad parece ser la dimensión a la que apelan los programas sociales para comenzar a definir sus destinatarios/ as. Ahora bien, las edades son arbitrarias: se refieren a una de las formas en que se representa la lucha por el poder y el acceso a recursos materiales y simbólicos, en este caso, entre unas personas más jóvenes y otras más viejas. A partir de esas luchas resultarán las fronteras, provisorias, que intentarán establecer el mantenimiento del orden y la eventual transformación (Bourdieu 1990). La edad es, por tanto, una categoría relacional. Cuando las edades biológicas se cruzan con condiciones de clase, con desiguales accesos a bienes materiales y simbólicos, y con pertenencia étnicas y de género, las historias que se configuran pueden resultar verdaderamente disimiles. De allí que resulte más útil definir a las etapas vitales como construcciones flexibles y que se constituyen y moldean en cada persona a partir de la interrelación de múltiples dimensiones y factores. Una mujer de 18 años puede ocupar una posición social muy distinta a la de un varón de la misma edad biológica, aún cuando pertenezcan a la misma clase social. La diferencia es lo que esa edad significa en cada contexto específico. Dichas posiciones, son creadas por, y crean, relaciones diferenciales con el Estado.

En efecto, así como la definición y el procesamiento de las edades son relacionales, la dimensión el género, como construcción social de la diferencia sexual, también lo es. Eleonor Faur (2004) sugiere que la tensión entre la elección individual de los sujetos y la coerción social del entorno es una de las que permite pensar la construcción social del gé- nero, por ejemplo, dentro de programas sociales que nunca son neutrales al respecto. Los programas actúan desde posiciones de género dado que sus trabajadores/as son sujetos imbuidos en el género, tienen sus percepciones sobre modelos deseables de ser varones o chicas jóvenes, y bajo esas ideas interpretan los planteos institucionales. En palabras de Scott (2000) las instituciones encarnan algunas, y no otras, de las representaciones circulantes sobre lo masculino y lo femenino como regulaciones normativas.

En suma, el Estado, y los programas sociales como manifestaciones concretas de su accionar, inciden con sus nominaciones y propuestas en las relaciones entre jóvenes $y$ adultos, y entre hombres y mujeres, modelando relaciones deseables de género y edad. A su vez, el Estado es capaz de desplegar distintos mensajes sobre estas relaciones qué, lejos de ser unívocos, varían de acuerdo a sus contextos específicos de enunciación, en los cuales las interpretaciones que hacen los agentes estatales se vuelven piezas centrales (Haney 1996; MacKim 2008). Al intentar convertirse en beneficiarias, las personas pondrán en discusión sus modos de vida cotidiana ${ }^{5}$ con las expectativas institucionales que existen sobre ellas, en función de su posición de clase, género o edad social. Si bien la discusión que mantienen ambas partes no supone unas prácticas equipotenciales, existe un espacio de contienda (Fraser 1991) en el que grupos dominantes y subordinados negocian las condiciones de la asistencia. Esto sucede, a su vez, porque el Estado habilita espacios de maniobra (Haney 2002) dentro de cuyos límites los/as beneficiarios/as pueden desplegar algunas contestaciones. Este artículo propone recuperar ese proceso negociado de construcción de feminidades al interior de un programa de prevención del delito, y ahondar en las consecuencias del mismo.

Materiales y métodos. Se analizan datos producidos en una investigación cualitativa sobre los modos de gobernar a la "juventud en riesgo" a partir de programas de prevención del delito. Específicamente se realizó un estudio de caso sobre una implementación del programa Comunidades Vulnerables, en un barrio del Gran Buenos Aires, Argentina, entre 2007 y 2011. La dinámica del Programa suponía reuniones semanales de 2 horas entre el equipo técnico y los/as jóvenes en distintos espacios del barrio. Se trataban temas en torno a 4 ejes: "vincular" (relacionado con el proyecto de vida), el "mundo del trabajo" (reinserción escolar y/ laboral), "jurídico" (fortalecimiento de derechos y resolución de causas penales), y "sociocomunitario". El Programa incluía una transferencia condicionada de ingresos (TCl) a la que los/as beneficiarios/as accedían si asistían a las reuniones y encaraban acciones tendientes a la elaboración de un proyecto de vida alternativo al delito, por ejemplo, retomar la escuela, conseguir un trabajo, tratar su adicción a las drogas, resolver su situación irregular frente a la justicia, etc. Durante el período en que se obtuvieron los datos el grupo de beneficiarios/as se componía de 31 varones y 15 mujeres todos/as argentinos/as, y el promedio de edad grupal era 22 años. Todas las chicas eran madres o estaban embarazadas y sólo un tercio de los varones tenían hijos/as (aunque no todos los tenían a cargo). Algunos de los beneficiarios/as habían co- 
metido delitos, otros/as tenían prácticas delictivas o estaban, según criterios del programa, en riesgo de hacerlo. El equipo técnico estaba compuesto por dos agentes estatales, una trabajadora social y un estudiante de comunicación social, y por una operadora comunitaria.

Se analizaron registros de campos de observación participante de 76 encuentros, testimonios de entrevistas en profundidad a jóvenes y a agentes estatales, y documentos institucionales. ${ }^{6}$ En los materiales se rastrearon: condiciones de acceso, permanencia y egreso del programa, expectativas sobre los impactos del programa en la vida de las y los jóvenes, logros y dificultades en la obtención de los resultados previstos, instancias de negociación entre oferta y demanda y consecuencias de las negociaciones entre partes.

\section{Resultados}

\section{Las definiciones institu-} cionales sobre la población beneficiaria. Los/as potenciales beneficiarios/as de este programa de prevención del delito serían adolescentes y jóvenes de entre 13 y 25 años que vivieran en situación de vulnerabilidad social: "en conflicto con la ley o en riesgo de estarlo: haber infringido una norma del código penal y no estar en situación de encierro; estar o haber estado involucrado en prácticas violentas próximas al delito; haber estado detenido en comisarías, institutos de menores $y / 0$ instituciones penitenciarias; haberse visto involucrado en detenciones preventivas en comisarías" (documento institucional, Dirección Nacional de Política Criminal, 2007). Complementariamente, en palabras de la agente estatal que implementaba el caso estudiado, lo que determinaba que ciertos/as jóvenes fueran beneficiarios/as era:

El grupo en riesgo, (para) trabajar la cuestión primaria de prevención del delito. Son pibes que capaz están cartoneando, juntándose con los de la esquina, la mitad de la familia delinquió, y viene la madre o un pariente o ellos mismos, por la motivación a salir a robar. O están en pequeñas acciones, y capaz no tienen causas, no cometieron delitos grandes, pero están en pequeñas actividades, o que afanan guita en la casa, o que participa como cómplice de campana en alguna situación delictiva pero todavía no se metió y está ahí.

Evidentemente, "la" situación de riesgo, podía ser muchas cosas. Es justamente la ambigüedad que recubre el término riesgo lo que lo hace tan fértil para la justificación de intervenciones estatales sobre cualquier aspecto de la vida que puede tener, potencialmente, consecuencias indeseables (Castel 2004; De Almeida Filho 2000). La amplitud del contenido del riesgo circundante, sumado a lo arbitrario de las edades, requería más precisiones.

Cuando entrevisté a la agente aclaró que se consideraba jóvenes en riesgo a quienes carecían de un proyecto de vida, ya que aún no lo habían podido armar. El Programa se orientaba a que los jóvenes construyeran un proyecto de vida alternativo al delito, como parte de la transición a la adultez.

Estas definiciones institucionales son complejas y enfatizan el procesamiento social de la dimensión etaria al tiempo que dan cuenta de su cruce con la posición de clase -desaventajada o vulnerable-. Sin embargo, nada se menciona sobre el género. En primer lugar el dato es llamativo porque según su creador, el Programa había sido pensado para varones, con lo cual sería esperable que alguna mención al sesgo se hiciera. Pero la sorpresa fue aún mayor cuando el silencio respecto del género continuaba aún cuando, en la implementación estudiada, la proporción de mujeres beneficiarias (y además madres) era considerable. El proyecto de vida alternativo o el vínculo con el riesgo, ¿era el mismo para chicas y chicos que compartían el rango etáreo? Al respecto, pregunté a la agente estatal si el Programa preveía admitir chicas que no hubieran cometido delitos pero que al ser madres estuvieran en situación de vulnerabilidad.

No, el perfil es el mismo, o que estén en riesgo, el marido, el cuñado, están todos presos ella está sola, y no tiene ingresos en la casa y está empezando a participar de alguna manera en la dinámica delictiva del barrio. Por eso se la incorpora, aunque no haya cometido delitos.

El estar "solas" -carecer de la compañía de un varón proveedor de ingresos- ponía a las chicas en riesgo respecto del delito. Su riesgo era estar "solas" de hombres; ni madres, hermanas, amigas, o ellas mismas podrían compensar esa carencia. Tal como describí en otro trabajo (Medan 2011) el riesgo de los varones no era la ausencia de chicas, sino la carencia de ingresos o mala influencia de pares. Sin embargo, esta posición "en riesgo" de ellas, habilitaba, al menos enunciativamente, el delito femenino.

No obstante, la mayoría de las beneficiarias no tenían explícitas relaciones con el delito, pero sí hijos. ${ }^{7}$ Esta situación me instó a preguntar ¿qué relaciones son posibles entre el delito y las chicas jóvenes?, ¿cómo 
se delinea el proyecto de vida para ellas?, ¿qué condiciones habilitan su transición a la adultez? y finalmente, el riesgo que las aquejaba y que legitimaba la intervención institucional ¿era el delinquir u otra cosa?

\section{La legitimación estatal de} las necesidades de las mujeres y sus consecuencias. De las 15 beneficiarias que tuvo el Programa durante los dos años de mi trabajo de campo, sólo 3 tenían explícitas relaciones con el delito (una había estado en prisión, y dos estaban imputadas y esperaban el juicio oral). El resto se presentaron argumentando necesidades materiales y dificultades de otras índoles vinculadas al cuidado de sus hijos. Cuando entrevisté a la operadora comunitaria, me explicó que el delito femenino estaba menos legitimado que el masculino:

Hay una cuestión de aceptación, porque creo que hay muchas mujeres que delinquen en el barrio pero hay más aceptación de que lo haga el varón a que lo haga la mujer, entonces creo que también se juega la vergüenza..

El delito femenino podía identificarse, pero no gozaba de la visibilidad ni legitimidad del masculino. Quizás por eso en la instancia de admisión al Programa, el delito femenino no formaba parte de la mayoría de los relatos. Si bien no es posible discernir si las chicas evitaban sus eventuales vínculos con el delito porque estaba "mal visto" o porque otras argumentaciones les garantizaban la misma ayuda con menos descrédito, lo cierto es que independientemente de sus relaciones con el delito, elegían presentarse como madres.

Valeria tenía 19 años y estaba embarazada cuando intentó convertirse en beneficiaria del Programa. Al entrevistarla le pregunté qué les había dicho a las agentes sobre su situación:

Valeria: Les dije que yo estaba peleada, no tenía a nadie.

Entrevistadora: ¿Con quién estabas peleada?

Valeria: Con el papá de la nena, por boludeces (...) la que me tomó la entrevista me dijo si yo estaba soltera y le dije que sí, y que después de tener la nena iba a buscar un trabajo. Ellas me dijeron que me iban a aguantar a que yo consiguiera un trabajo.

Valeria representaba el típico caso de riesgo que el Programa preveía considerar para justificar la atención de las chicas: ella estaba en riesgo porque estaba "sola". Esa condición bastaba y no requería ahondarse en si además existían o no vínculos con el delito.

Leticia, de 25 años y con una hija de 4, ilustra otro caso típico. Esta beneficiaria, luego de haberse ausentado unos meses sin explicaciones -tras lo que se le suspendió la TCl-, se presentó un día a la reunión semanal. Entonces, le contó a la agente estatal que se le había terminado el trabajo temporal que tenía y que, aunque con los ingresos que traía el marido de trabajos esporádicos se arreglaban, ella podía volver al Programa y consecuentemente, volver a cobrar la TCl. Además anunció que estaba nuevamente embarazada y la ayuda económica colaboraría con los gastos de la familia ampliada. Sin mediar ninguna charla sobre el compromiso personal que ella pensaba adquirir con el Programa para convertirse nuevamente en beneficiaria, se le volvió a dar de alta dentro del grupo de beneficiarios/as.

Sin delitos asociados y con un marido que trabajaba, ¿qué necesidad de Leticia la convertía en una legítima merecedora de la ayuda estatal? Al tener marido ni siquiera estaba "sola" en los términos en que el Programa definía la situación de riesgo de las chicas. Este ejemplo insinúa que la "soledad" de las chicas podría estar más asociada a una condición "esencial" de las madres que viven en contextos desaventajados, que a la compañía o no de un varón de carne y hueso. La condición materna no sólo les permitía ser admitidas en el Programa sino también permanecer en él, exceptuándolas de las contraprestaciones que el dispositivo tenía previstas para sus beneficiarios/as por cobrar la TCl (estudiar, capacitarse en oficios, regularizar situaciones judiciales, tratar adicciones, etc.). Para las chicas, cuidar de sus hijos parecía ser un compromiso válido no enunciado formalmente.

Silvina, una de las beneficiarias, parecía tenerlo bien en claro. Ella, de 28 años y madre de 6 niños/as, aceptó volver a la escuela como contrapartida de la ayuda económica recibida. Lo haría en horario nocturno y llevaría a su hija más pequeña con ella porque no tenía con quien dejarla. Si bien al principio las maestras no comprendían la presencia de la chiquita en el aula, luego la aceptaron, y Silvina estaba contenta. Sin embargo, dos meses más tarde, ya no iba más a la escuela. Al explicar la deserción a la agente del Programa, le contó que estaba nuevamente embarazada. Además, ir a la escuela de noche implicaba llegar muy tarde a la casa, que su marido al llegar del trabajo no tuviera la cena preparada, y que incluso sus hijos más pequeños se quedaran dormidos antes de comer. Si bien al dejar la escuela Silvina incumpliría el compromiso personal que había 
asumido para ser beneficiaria, sus razones fueron entendidas como válidas. Parecía lógico, a los ojos del Programa, que con varios chicos a cargo y un embarazo a cuestas, la escuela implicara demasiado esfuerzo. Silvina recibió felicitaciones por el nuevo embarazo y siguió "contraprestando" a través de su rol de madre y buena esposa.

En efecto, la condición solitaria y materna era esgrimida por las chicas como pedido de morigeración para comportamientos que en otros casos serían sancionados. Una de las jóvenes narraba cómo negoció el reingreso luego de haber sido "dada de baja" alrededor de su condición materna:

- [la baja se produjo] porque falté, pero fijate que yo tenía a Ramiro $y$ teníamos las reuniones a las 10 de la mañana, un mes falté y fui y le dije, entiéndame acabo de salir de estar presa, que el bebé tiene meses

\section{- Estaban en invierno}

- Claro, vivía en un casa que no es como la de ahora que está todo cerrado y no entra frío ni nada, no estaba su papá para que lo cuide él o su hermana, tenía que estar sí o sí yo con él y yo tenía que darle la teta.

- ¿Y no lo llevabas al programa?

- No, después sí lo llevé cuando se fue el invierno.

- Y ¿qué pasó?

- Y me entendieron y después al mes me dieron el alta [nuevamente] (Bibiana, beneficiaria).

Del delito, en un programa que intentaba prevenirlo, pocas noticias. Estos casos inducen a pensar que las beneficiarias consideran a los programas como una suerte de compensación económica por cuidar a sus hijos. A su vez, la persistencia de ellas -y sus particulares situaciones- en este tipo de dispositivos, demuestran que éstos coinciden con, o incluso han contribuido a crear, esta interpretación de las beneficiarias.

Discusión. En un trabajo anterior (Medan 2013b) propuse una tipología de riesgos que, según los programas, afectarían a los y las jóvenes. El primer tipo reuniría a los riesgos "externos": aquellos que surgen en instancias externas al sujeto -por ejemplo, el hostigamiento policial hacia jóvenes pobres-; el segundo, referido a riesgos "mixtos", de origen externo pero con permeabilidad muy alta hacia el sujeto, por su propia predisposición a dicha influencia: por ejemplo, influencias familiares o comunitarias del entorno; y finalmente un tercer tipo, los "esenciales" vinculados con la dimensión de género. En ellos quiero detenerme.

Las narrativas del riesgo tienen marcas de género. Los vínculos con el delito y el uso inapropiado de la fuerza física son riesgos asociados a los varones quienes parecen más amenazados por las malas "juntas", y por la necesidad de conseguir ingresos extras. Los varones delinquirían por una relación que aparece como "natural" -en tanto no problematizada- entre su exposición al riesgo como modo de socialización y su constitución como varones (Medan 2011). Siguiendo este diagnóstico de riesgos, el proyecto de vida alternativo que los programas sugieren para los beneficiarios propicia un modelo de varón proveedor que obtenga ingresos legalmente, evite comportamientos riesgosos, irracionales e infantiles como el delito y sus acciones asociadas, y que privilegie oportunidades de capacitación para proyectar un futuro deseable. En base a este modelo, se refuerzan estereotipos de masculinidad al no asumir como construidas estas relaciones y dejarlas sin problematizar. Mientras que la disputa respecto de los mandatos de género queda invisibilizada, aquella en relación a la dimensión generacional es más cruda. El Programa reprende a los varones calificando sus acciones como "rebeldes" y los insta a "madurar". En este sentido, sostengo que los coloca en una posición de subordinación, infantilizándolos, al tiempo que se ubica en la vereda de la adultez para legitimar el modelo de transición masculina deseable que propone.

Ahora bien, como se anticipó, el riesgo que aqueja a las chicas es el "estar solas" y el Programa, más que discutir en contra de esta situación -como hace con los varones y sus estilos de vida- parece reconocerla como merecedora de asistencia. La jerarquía que las mismas mujeres jóvenes dan a la maternidad, al interponerla como una de las variables principales para requerir asistencia, constituye para el Programa una señal de madurez de parte de ellas. La maternidad se configura como un proyecto de vida y de inclusión que las protege de los "desvíos" hacia el delito. Esta situación redunda en que las mujeres sean objeto de menos control duro que los varones. A ellas no se les pide madurar, ni estudiar, ni trabajar, porque -lógicamentebastantes tareas tienen con sus hijos/as. Las chicas, según este esquema, habrían completado una transición generacional deseable al momento de convertirse en madres, dejando atrás la juventud descarriada y problemática. El conflicto generacional, entre la institución (adulta) que se propondría transformar y conducir la transición generacional de los/as jóvenes, perdería relevancia. En suma, 
las beneficiarias parecían más dóciles que los varones.

Ahora bien, la mirada aprobatoria que el Programa tenía sobre las mujeres no estaba exenta de consecuencias: condicionaría la configuración de feminidades y restringiría aspectos de la experiencia juvenil y femenina. La contracara de la "ventaja" que tendrían las chicas al orientarse de acuerdo al modelo institucional sería que éste las situaría definitivamente en un lugar de vulnerabilidad. Esto porque no sólo requerirían ayuda para sostener su propia vida sino que además, al tener otros sujetos a cargo y carecer de un varón, deberían aceptar la ayuda estatal con las condiciones que ésta les impusiera. Además, las confinaría a esos espacios domésticos, no remunerados y menos jerarquizados que los que proponía a los varones. Así, el Programa adhería a una mirada tradicional indicando que las mujeres deben permanecer al cuidado de sus hijos. La validación de la maternidad que se advirtió en el trabajo de campo obstruía -por omisión- otras posibilidades de conciliar la feminidad, la familia y la obtención de ingresos propios. Además, al asistirlas porque estaban solas reforzaba la idea de que las mujeres se definen en base a sus relaciones con hombres, como esposas, madres, hijas o hermanas; en contraste con lo que ocurre con las distinciones correspondientes para los hombres (Ortner y Whitehead 2000). Pero por otro lado, y más sutilmente, su condición de "soledad" parecía intrínseca a su condición de madres pobres porque el Programa las asistía aún cuando tenían parejas: siempre las reconocía vulnerables y cumplía el rol proveedor que no adoptaban esos varones, o que no hacían bien.
Este procedimiento las señalaba como mujeres carentes, siempre necesitadas de ayuda de alguien o algo que se colocara en la posición de varón proveedor. En síntesis, las chicas jóvenes merecen ayuda, no por ser "rebeldes" y persistir en los errores -como los varones-, sino por ser "débiles". Finalmente, se invisibilizaba, por contraste con la notoriedad de la maternidad, la relación de las chicas con el delito. $Y$ tal como sugiere Elizalde (2013:57) habrá que preguntarse si esa invisibilidad constituye un dato de la realidad pasible de verificación o de "un efecto de lectura de una construcción ideológica específica que 'borra' a las mujeres de cierto ámbito de la 'peligrosidad' para fijarlas en el campo de la 'desviación sexual', la 'vulnerabilidad' y el 'riesgo social', y operar, desde allí, una regulación diferencial de sus prácticas". La operación tiene un doble resultado. Como el género es un sistema de diferencias, al confinar a las chicas a ámbitos privados, mostrarlas alejadas de las prácticas delictivas o arriesgadas y menospreciar su capacidad disruptiva, habilita que los varones ocupen esos lugares. Esto contribuye, a reforzar los estereotipos que los vinculan naturalmente con dichas acciones. Así, no sólo se configuran feminidades, sino también masculinidades.

A pesar de estos efectos de subordinación, las jóvenes reconocen las asociaciones tradicionales entre feminidad y vulnerabilidad, e -independientemente de que se sientan reflejadas-, hacen un uso estratégico de esta "debilidad", y se presentan en nombre de dicha interpelación. Apelan tanto a una "hibridez de los enunciados, que combinan la interpretación propia con un discurso institucional" (Llobet 2009:250), como a su capacidad de producir respuestas subalternas a las interpelaciones institucionales, aún desde posiciones de subordinación. Posiblemente, como fruto de sus anteriores vínculos con la "ayuda" estatal, las chicas han aprendido a presentarse como merecedoras de asistencia en pos del cuidado de sus hijos/as. Aunque la utilización estratégica que las chicas pueden hacer de su maternidad no logra neutralizar, ni mucho menos subvertir, su multideterminada posición subordinada, este efecto no parece contrariarlas. Más aún, inspirándome en una análisis empírico de Haney (1996), podría suponerse que si acaso, en un giro aparentemente progresista de la política -comprometido con la búsqueda de mayor autonomía para las mujeres y con la deconstrucción de los estereotipos que las asocian naturalmente a la maternidaddejara de brindarse ayuda a estas jóvenes por presentarse como madres, ellas verían esta operación, mucho más como una injusticia que como un reconocimiento de sus necesidades.

\section{Consideraciones finales.}

Como parte de su tarea de control y garantía de la reproducción social, los programas sociales propugnan transiciones legítimas e ilegítimas hacia la adultez diferenciadas según género. Atender a los cruces entre la edad social y el género en la construcción de beneficiarios y beneficiarias no sólo supone enfocar en el futuro -en tanto preocupación por las transiciones-. También permite enfocar en cómo se configuran identidades de género y edad en el presente, que impactan en las vidas cotidianas de los y las jóvenes. Aquí quise exponer un análisis sobre ambos procesos. Las chicas transitarán correcta- 
mente a la adultez, vinculadas a su proyecto de maternidad, que las incluye socialmente pero desde una posición subordinada a la ayuda masculina o estatal. A su vez, ese mismo proyecto les sugiere modelarse según una versión femenina adulta que limita -en la mayoría de los casos- sus capacidades disruptivas y sus despliegues públicos y autónomos. Ellas, más o me- nos estratégicamente, prestan complicidad a la propuesta, y parecen obtener beneficios sin mostrarse contrariadas por las posiciones que dicho acceso les tiene reservadas.

Notas:

* Este trabajo retoma argumentos sostenidos en un trabajo anterior (Medan, 2013a).

** Doctora de la Universidad de Buenos Aires (UBA) en Ciencias Sociales. Investigadora asistente del Consejo Nacional de Investigaciones Científicas y Tecnológicas de Argentina e investigadora del Centro de Estudios Desigualdades, Sujetos e Instituciones de la Universidad Nacional de General San Martín. Correo electrónico: marinamedan@gmail.com

1. Este artículo entiende a los programas estatales como dispositivos que representan y exceden al Estado. Por un lado, porque el Estado al que representan es entendido como una entidad multifacética y conformada en capas, compuesta por diferentes aparatos que pueden operar de forma inconsistente (Haney, 1996). Los programas, a su vez, lo exceden ya que logran su forma final en su implementación localizadas en espacios y tiempos específicos, en las que sus diseños programáticos entran en interacción cotidiana, mediados por agentes estatales, con sus beneficiarios/as.

2. Con respecto a esta última dimensión, el género, las definiciones de destinatarios suelen no pronunciarse; esto supone que -en principio-admiten chicas y varones; en algunos pocos casos hay programas destinados a mujeres jóvenes -en general vinculados con temas de salud sexual y reproductiva-, y no tenemos constancia de que existan programas destinados sólo a varones.

3. La focalización en la generación y el género no supone invisibilizar los condicionantes de clase o étnicos. La condición socioeconómica desaventajada a la que pertenecen los/as jóvenes que protagonizan el análisis atraviesa el mismo. Sobre este tema pueden consultarse Medan (2012). La condición étnica, en este estudio, no se presentó como una variable significativa.

4. Lo "deseable" de las transiciones hace referencia no sólo a la motivación de gestar trayectorias vitales que se incorporen activamente a la vida en la sociedad, sino también, y sobre todo, a evitar trayectorias "indeseables" que atenten contra el orden social. En este último sentido de trata más de gestionar la marginalidad que de tender hacia una efectiva inclusión social.

5. Como se señaló, esos modos de vida no son configurados de forma autónoma, sino, de forma relacional, en la medida en que las personas nacen, se crían y crecen en el seno de instituciones (la familia, el mercado, la escuela, los medios de comunicación, etc.) cuyas ideas sobre la edad y el género permean sus vidas.

6. Se han cambiado los nombres de los informantes para proteger su identidad.

7. Según los testimonios y materiales institucionales, desde el inicio de la implementación en 2001 hasta 2008, el promedio de chicas era de 1 cada 7 varones. Desde 2008, la proporción aumentó y se registraron promedios de 1 chica cada 3 varones. 


\section{Bibliografía:}

BOURDIEU, Pierre (1990). Sociología y cultura, Grijalbo-CNCA, México.

CASTEL, Robert (2004). La inseguridad social. ¿Qué es estar protegido?, Manantial, Buenos Aires.

CHAVES, Mariana (2005). "Juventud negada y negativizada: representaciones y formaciones discursivas vigentes en la Argentina contemporánea" en Última Década, año 13, № 23, 9-32.

DE ALMEIDA-FIHLO, Naomar (2000). La ciencia tímida. Ensayos de deconstrucción de la epidemiología, Lugar Editorial, Buenos Aires.

Dirección Nacional de Política Criminal (DNPC) (2007). Plan Nacional de Prevención del delito. Programa Comunidades Vulnerables, COGCAL, Buenos Aires.

ELIZALDE, Silvia (2013). La otra mitad. Género y pobreza en la experiencia de mujeres jóvenes, EdUNLP, La Plata.

FAUR, Eleonor (2004). Masculinidades y desarrollo social. Las relaciones de género desde las perspectivas de los hombres, UNICEF/Arango, Bogotá.

FRASER, Nancy. (1991). "La lucha por las necesidades: Esbozo de una teoría crítica socialista-feminista de la cultura política del capitalismo tardío", en Debate Feminista, Vol. 2, №3.

HANEY, Linne (1996). "Homeboys, babies, men in suits: the satate and the reproduction of male dominance" en American Sociological Review, $N^{\circ} 5$.

HANEY, Linne (2002). Inventing the needy: gender and the politics of welfare in Hungary, University of California Press, Berkeley.

LLOBET, Valeria (2009). ¿Fábricas de niños? Las instituciones en la era de la infancia, Novedades educativas, Buenos Aires.

MCKIM, Alice. (2008). "'Getting gut-level': Punishment, Gender, and Therepeutic Governance" en Gender and Society, Vol. 22, № 3, 303-323.

MEDAN, Marina (2011). "Sociabilidad juvenil masculina y riesgo: Discrepancias y acuerdos entre un programa de prevención del delito juvenil y sus beneficiarios" en Última Década, vol. 19, №35, 61-87.

MEDAN, Marina. (2013a). "Prevención del delito y construcción de feminidades juveniles" Revista de Ciencias Sociales, № 140 (II), 73-83.

MEDAN, Marina (2013b). "Los sentidos del riesgo en programas para jóvenes". En LLOBET, Valeria. Sentidos de la exclusión social. Beneficiarios, necesidades y prácticas en políticas sociales para la inclusión de niños y jóvenes en el área metropolitana bonaerense, Biblos, Buenos Aires.

ORTNER, Sherry y WHITEHEAD, Harriet (2000). "Indagaciones acerca de los significados sexuales”. En LAMAS, Marta (Comp.), El género. La construcción cultural de la diferencia sexual, PUEG, México.

SCOTT, Joan (2000). "El género: una categoría útil para el análisis histórico". En LAMAS, Marta (comp.), El género. La construcción cultural de la diferencia sexual, PUEG, México. 